\title{
MiR-338-3p improved lung adenocarcinoma by AKAP12 suppression
}

\author{
Jin Chang' ${ }^{1}$, Shuo Liư ${ }^{2}$ Baowei Li ${ }^{3}$ Zhongchao Huo ${ }^{4}$ Xiaomin Wang ${ }^{3}$, Hui Zhang ${ }^{3}$
}

\author{
1'Department of Aesthetic Surgery, Affiliated Hospital of Hebei University \\ of Engineering, Handan, Hebei, China \\ 2Department of Stomatology, Handan First Hospital, Handan, Hebei, China \\ ${ }^{3}$ Department of Radiology, Affiliated Hospital of Hebei University of Engineering, \\ Handan, Hebei, China \\ ${ }^{4}$ Department of Radiotherapy, Affiliated Hospital of Hebei University of Engineering, \\ Handan, Hebei, China
}

Submitted: 15 September 2017; Accepted: 1 December 2017; Online publication: 19 December 2019

Arch Med Sci 2021; 17 (2): 462-473

DOI: https://doi.org/10.5114/aoms.2019.90913

Copyright $\odot 2019$ Termedia \& Banach

\section{Abstract}

Introduction: This study aimed to explore the biological functions of AKAP12 in lung adenocarcinoma and investigate the interaction between AKAP12 and miR-338-3p.

Material and methods: Sixty-one differentially expressed genes in lung adenocarcinoma and adjacent normal tissues were first analyzed by TCGA. Immunohistochemistry and quantitative reverse transcription PCR (qRT-PCR) were further utilized to confirm aberrant $A K A P 12$ expression in tumor tissues. The influences of $A K A P 12$ on proliferation, invasion and migration, and apoptosis of lung adenocarcinoma were investigated by clone formation assay and MTT assay, transwell assay, and flow cytometry analysis respectively. TargetScan and miRanda databases predicted the binding sites of miRNAs on AKAP12 3'-UTR and structure changes were validated by RNA folding form. The target relationship between miR-338-3p and AKAP12 was confirmed by the dual-luciferase reporter system. Disease-free survival (DFS) and overall survival (OS) curves were generated with Kaplan-Meier plotter according to the TCGA data and the correlation among AKAP12 expression, miR-338-3p expression and prognosis was also analyzed.

Results: AKAP12 was upregulated in lung adenocarcinoma tissues and cells (all $p<0.01$ ), and negatively correlated with prognosis outcomes of patients (both $p<0.05$ ). High expression of AKAP12 promoted proliferation, invasion and migration of cancer cells, and inhibited cell apoptosis (all $p<$ 0.05). MiR-338-3p could directly bind to the 3'-UTR of AKAP12 and showed most significant suppression on AKAP12 expression among four predicted miRNAs (all $p<0.01$ ). Additionally, miR-338-3p could suppress AKAP12 in lung adenocarcinoma, improving prognosis (all $p<0.05$ ).

Conclusions: AKAP12 acts as a tumor promoter in lung adenocarcinoma development. Upregulation of MiR-338-3p could suppress AKAP12 expression in lung cancer cells and contribute to a better prognosis.

Key words: lung adenocarcinoma, AKAP12, miR-338-3p.

\author{
Corresponding author: \\ Hui Zhang \\ Department of Radiology \\ Affiliated Hospital of Hebei \\ University of Engineering \\ 81 Congtai Road \\ Handan 056002 \\ Hebei, China \\ Phone: +86 0310-8572101 \\ E-mail: \\ wushuming2007@126.com
}

\section{Introduction}

Lung cancer, predominantly non-small-cell lung cancer (NSCLC), is the chief culprit for cancer mortality [1]. It is characterized by progressive metastasis and poor prognosis [2]. It is reported that lung cancer accounts for 
more than one-quarter (26\%) of all cancer deaths, and the 5 -year survival of lung cancer is only $18 \%$ [3]. Over the past decades, targeted therapeutics have been developed against drivers of lung adenocarcinoma, a subtype of NSCLC, improving the prognosis of patients [4]. Therefore, it is essential to further explore the underlying mechanisms of lung adenocarcinoma development and develop more efficient targeted treatment.

MiRNAs widely involved in cellular processes via bind to the $3^{\prime}$-untranslated region (3'-UTR) of genes and regulate cell functions $[5,6]$. MiR-338-3p, located on chromosome $17 q 25$, is a miRNA which plays a critical role in promoting cell death, neuronal differentiation and neurite extension [7]. Accumulating evidence has indicated that miR-338-3p could suppress the development of various human cancers, including hepatocellular carcinoma [8], breast cancer [9] and lung cancer [10]. It plays an anti-tumor role in lung cancer, inhibiting proliferation, migration, invasion, enhancing cell apoptosis [11] and suppressing tumor metastasis $[12,13]$. Several studies have reported that several oncogenes were downregulated by miR-338-3p in lung cancer, such as IRS2 [10], SphK2 [14] and SOX4 [15]. Further investigation was needed to find more related genes for better understanding of lung cancer development.

AKAP12 is an A-kinase scaffold protein that possesses a characteristic binding domain for the regulatory subunit of protein kinase $A[16,17]$. The loss of $A K A P 12$ is associated with increased cancer susceptibility [16]. AKAP12 functions differently in various types of cancers. Xia et al. found that AKAP12 was inhibited by miR-103 in hepatocellular carcinoma, thus promoting tumor growth [18]. However, other studies suggested that overexpression of AKAP12 predicted a poor prognosis and promoted the tumor development in ovarian cancer [19] and melanoma [20]. Although evidence has highlighted that expression of AKAP12 was closely related to cancer development, current studies on its biological function in lung cancer are limited, and the interaction between miR-338-3p and $A K A P 12$ also has not been determined.

In the present study, we investigated whether $A K A P 12$ influenced the proliferation, migration, invasion and apoptosis in lung adenocarcinoma, and examined the relationship between AKAP12 and miR-338-3p. Our results suggested that miR-338-3p improved the prognosis of lung adenocarcinoma by suppressing AKAP12, providing a potential target for clinical treatment of lung adenocarcinoma.

\section{Material and methods}

\section{Human specimens}

In this study, 20 paired human lung adenocarcinoma tissues and adjacent non-tumor ones were obtained from the Affiliated Hospital of Hebei University of Engineering Hospital (Handan, China). No tissues were gained from patients who had received preoperative radiotherapy or chemotherapy before. Specimens were immediately frozen in liquid nitrogen and stored at $-80^{\circ} \mathrm{C}$ until usage. The ethics committee of the Affiliated Hospital of Hebei University of Engineering approved this study.

\section{Cell line and cell culture}

Lung adenocarcinoma cell lines A549 and H1299 and normal human epithelial cell line HBE were purchased from BeNa Culture Collection (Beijing, China). Lung adenocarcinoma cell line A549 and normal cell line HBE were cultured in 90\% Dulbecco's modified eagle medium (DMEM, Sigma, St. Louis, MO, USA) with high glucose supplemented with $10 \%$ FBS, while H1299 was maintained in Roswell Park Memorial Institute (RPMI) 1640 medium (Sigma) with 10\% FBS. Cells were all cultured in $5 \% \mathrm{CO}_{2}$ at $37^{\circ} \mathrm{C}$.

\section{Bioinformatics analysis}

Microarray analysis was performed with the TCGA database, and 58 adjacent tissues and 58 tumor tissues in lung adenocarcinoma were involved respectively. Differentially expressing genes were recognized by an adjusted $p$-value less than 0.05 and $\log _{2}$ fold change larger than 10 or less than -10 . After being ranked, the ten most highly expressed genes and most lowly expressed genes were listed in Supplementary Table SI. The heat map and volcano plot were drawn with $\mathrm{R}$ studio.

TargetScan and miRanda were used to predict the target relationship between AKAP12 and different miRNAs. Variable scores in the above two websites were recorded as shown in Supplementary Table SII: the mirSVR score in miRanda is an estimate of the miRNA effect on the mRNA expression level. The more negative the score, the greater the effect; the PhastCons score measures the conservation of nucleotide positions and the higher the score, the stronger the conservation; the Context++ score percentile indicated the percentage of sites for the miRNA with a less favorable context++ score, which is the sum of the contribution of 14 features [21], and a higher percentile means a greater combination probability.

RNA Folding Form website was used to predict the secondary structure of mRNAs. A greater structure change indicated a greater combination probability.

\section{Immunohistochemistry}

Lung adenocarcinoma tissue sections were firstly deparaffinized and rehydrated by ethanol 
solutions. After being washed by PBS 2-3 times, they were incubated with $3 \% \mathrm{H}_{2} \mathrm{O}_{2}$ for $10 \mathrm{~min}$ and then were washed again with PBS. Sections were then immersed in $0.01 \mathrm{M}$ citrate buffer ( $\mathrm{pH}$ 6.0), heated by microwave at $95^{\circ} \mathrm{C}$ for $20 \mathrm{~min}$, and cooled down at room temperature before cool water washing. They were then incubated with $3 \%$ goat serum sealant after PBS washing at room temperature for $20 \mathrm{~min}$. Sections were firstly incubated overnight with primary rabbit anti-human antibody, anti-AKAP12 (1 : 200, ab198895, Abcam, Cambridge, MA, USA) at $4^{\circ} \mathrm{C}$. Then they were washed in PBS and incubated with secondary antibody, goat anti-rabbit lgG-HRP $(1: 1000$, ab6721, Abcam) at room temperature for $30 \mathrm{~min}$. Following the final PBS washing, they were exposed to newly prepared diaminobenzidine for 4-6 min for $15 \mathrm{~s}$ of hematoxylin staining, in advance of dehydration by ethanol and air drying for observation. According to the positive staining intensity in immunohistochemistry assay, we set it to be that: colorless is score $0(-)$, pale yellow is score $1(+)$, palm yellow and above is score $2(++)$. Six fields were randomly chosen.

\section{Cell transfection}

NC mimics, miR-107 mimics, miR-613 mimics, miR-206 mimics, miR-338-3p mimics, AKAP12pcDNA3.1, sh-AKAP12-pcDNA3.1 and pcDNA3.1 plasmid vector were all obtained from GenePharma (Shanghai, China). Before transfection, cells were incubated in a 6 -well plate in $5 \% \mathrm{CO}_{2}$ at $37^{\circ} \mathrm{C}$ for $18-24 \mathrm{~h}$. Lung adenocarcinoma cells were firstly transfected with pcDNA3.1 plasmid vector as the control, AKAP12-pcDNA3.1 as the AKAP12 group, and sh-AKAP12-pcDNA3.1 as the sh-AKAP12 group, respectively. Cancer cells were also transfected with $100 \mathrm{nM} \mathrm{NC}$ mimics, miR107 mimics, miR-613 mimics, miR-206 mimics or miR-338-3p mimics respectively by Lipofectamine 2000 (Invitrogen, Carlsbad, CA, USA) according to the manufacturer's instructions. Lung adenocarcinoma cells comprised four groups: cells co-transfected with $50 \mathrm{nM} \mathrm{NC}$ mimics and $2 \mu \mathrm{g}$ of pcDNA3.1 plasmid vector were the control group, cells co-transfected with $50 \mathrm{nM} \mathrm{NC}$ mimics and $2 \mu \mathrm{g}$ of AKAP12-pcDNA3.1 were the AKAP12 group, cells co-transfected with $50 \mathrm{nM}$ miR-338-3p mimics and $2 \mu \mathrm{g}$ pcDNA3.1 plasmid vector were the miR-338-3p mimic group, and cells co-transfected with $2 \mu \mathrm{g}$ of AKAP12-pcDNA3.1 and $50 \mathrm{nM}$ miR338-3p mimics were the AKAP12 + miR-338-3p mimic group.

\section{Quantitative reverse transcription PCR}

Total RNA was extracted from cells by TRIzol reagent (Takara Bio, Inc., Otsu, Japan). Reverse transcription of extracted RNA and real-time PCR were performed using the Reverse Transcription Kit (Takara Bio) and miRNAs qPCR Quantitation Kit (Takara Bio) according to the instructions of the manufacturer, with GAPDH as an internal control.

Table I. Sequences for RT-qPCR, PCR, siRNA and mimics

\begin{tabular}{|lc|}
\hline Gene & Sequence (5' ${ }^{\prime}{ }^{\prime}$ ) \\
\hline q-AKAP12-F & CTGCCTTGGGAGTTGCC \\
\hline q-AKAP12-R & GGGTTACGCCTTCCCCAAG \\
\hline q-GAPDH-F & ACCACAGTCCATGCCATCA \\
\hline q-GAPDH-R & TCCACCACCCTGTTGCTGT \\
\hline AKAP12-cDNA-F & GCTAGCTAGAGAAGTGCGGAG \\
\hline AKAP12-CDNA-R & CTCGAGGTGGTACACATATGTC \\
\hline AKAP12 shRNA1 & CACCGCCAGAAGGAGCCCTGAACGAATTCAGGGCTCCTTTCTGGC \\
\hline AKAP12 shRNA2 & CACCGCTACTCACTGTGAAGAAACGAATTTCTTCACAGTGAGTAGC \\
\hline AKAP12 shRNA3 & CACCGCTGAAGAATTGGTTGAAACGAATTTCAACCAATTCTTCAGC \\
\hline shRNA control & CACCGCCGAACGAGTCCGAGAACGAATTCTCGGACTCGTTTCGGC \\
\hline miR-107 mimics & AGCAGCAUUGUACAGGGCUAUCA \\
\hline miR-613 mimics & AGGAAUGUUCCUUCUUUGCC \\
\hline miR-206 mimics & UGGAAUGUAAGGAAGUGUGUGG \\
\hline miR-338-3p mimics & UCCAGCAUCAGUGAUUUUGUUG \\
\hline Mimics control & UACGGGAAUUGCUUCGCGUG \\
\hline F- forward primer, $R-$ reverse primer. & \\
\hline
\end{tabular}


Data were analyzed by StepOne Software (Applied Biosystems, Foster City, CA, USA). The relative ex-

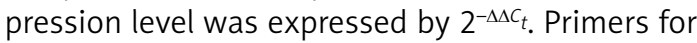
quantitative reverse transcription PCR (qRT-PCR) were synthesized by Huada Gene (Shenzhen, China) and listed in Table I.

\section{Western blot}

Total protein was extracted using ice-cold lysis buffer (Beyotime, Shanghai, China). Proteins were separated by SDS-PAGE with an electrophoresis system (Bio-Rad, Hercules, CA, USA), and then were transferred to polyvinylidene fluoride (PVDF) membrane (Invitrogen). After being blocked with 5\% skimmed milk for $4 \mathrm{~h}$, the PVDF membrane was incubated with primary rabbit anti-human antibodies, anti-AKAP12 (ab198895, 1 : 1000, Cambridge, MA, USA) and anti-GAPDH (ab9485, 1 : 2500, Cambridge, MA, USA). After incubation overnight at $4^{\circ} \mathrm{C}$, the membrane was then incubated with secondary antibody, goat anti-rabbit IgG-HRP (ab6721, 1 : 2000), for 1.5 h. Protein expression was then visualized by ECL Plus Western Blotting Substrate (Thermo Scientific, Waltham, MA, USA). The concentration of proteins was quantified by Quantity One Software (Bio-Rad).

\section{Clone formation assay}

Cells were seeded in plates (200-300 cells per plate) and incubated in $5 \% \mathrm{CO}_{2}$ at $37^{\circ} \mathrm{C}$ for $1-$ 2 weeks until cell colonies were visible. After PBS washing, they were fixed with $4 \%$ paraformaldehyde for $15 \mathrm{~min}$ and stained with $0.1 \%$ crystal violet for observation. Finally, clone number was quantified with a microscope.

\section{MTT assay}

Approximately $2 \times 10^{3}$ cells were seeded into 96-well plates for 3-5 days of incubation after suspension. Then $10 \mu$ of MTT ( $5 \mathrm{mg} / \mathrm{ml}$, Sigma) was added into each well for another $4 \mathrm{~h}$ of incubation. Supernatants were removed and $100 \mu \mathrm{l}$ of dimethyl sulfoxide (DMSO, Sigma) was added to each well for 10 min shaking. Then the absorbance (optical density) was measured at $490 \mathrm{~nm}$ on a microplate reader (Molecular Devices, Sunnyvale, CA, USA).

\section{Transwell assay}

The upper chamber of a transwell chamber (Costar, Corning Inc., Corning, NY, USA) was plated with approximately $1 \times 10^{5}$ cells without serum, while the lower chamber was plated with $800 \mu \mathrm{l}$ of culture medium (5 mg/l fibronectin and $10 \%$ FBS). After incubation at $37^{\circ} \mathrm{C}$ in $5 \% \mathrm{CO}_{2}$ for $24 \mathrm{~h}$, non-invading cells were removed from the upper chamber. Three independent fields were selected to observe hematoxylin and eosin stained bottom cells.

\section{Flow cytometry (FCM) analysis}

Transfected lung adenocarcinoma cells A549 and $\mathrm{H} 1299$ were incubated for $48 \mathrm{~h}$, and then stained with a FITC Annexin V Apoptosis Detection Kit (BD Biosciences, Bedford, MA, USA). Then cells were subjected to apoptosis detection by FACSCalibur FCM (BD Biosciences). The data were analyzed by the software FACS Diva (BD Biosciences).

\section{Dual-luciferase reporter system}

Plasmid vector psiCHECK2 was purchased from Promega (Madison, WI, USA). 3'UTR sequences of wild-type (WT) and mutant-type (MT) AKAP12 were inserted into the cleavage sites of the psiCHECK2 vector. PsiCHECK2-UTR vectors, negative mimics and miR-338-3p mimics were transfected into cancer cells using Lipofectamine 2000 (Invitrogen). Firefly and Renilla luciferase activities were measured $48 \mathrm{~h}$ later using the Dual-Luciferase Reporter System (Promega).

\section{Statistical analysis}

Data in this study were analyzed by GraphPad Prism 6.0 (Intuitive Software for Science, San Diego, CA, USA). Values were mean \pm standard deviation. Two groups were compared with Student's $t$ test, while multiple groups were compared using one-way ANOVA. All experiments were independently performed at least three times. The value of $p<0.05$ was considered statistically significant.

\section{Results}

\section{AKAP12 was highly expressed in lung adenocarcinoma}

According to the TCGA analysis, 61 genes in total were selected for their aberrant expression in lung adenocarcinoma tissues $(n=58)$ compared with normal tissues $(n=58)$. Then 10 high-expressed genes and 10 low-expressed genes were listed in Supplementary Table SI and heatmap and volcano plots were drawn in Figure 1 A. AKAP12, a confusing gene which functioned differently in different cancers, was found overexpressing in lung adenocarcinoma tissues. Analyzed with Kaplan-Meier method, high expression of AKAP12 contributed to worse prognosis regarding shorter disease free survival time (DFS) and overall survival time (OS) $(p<0.01$, Figure $1 \mathrm{~B})$. Immunohistochemical staining was performed in 20 paired lung carcinoma tissues and adjacent normal tissues. The staining value for each sample was presented in Supple- 
A
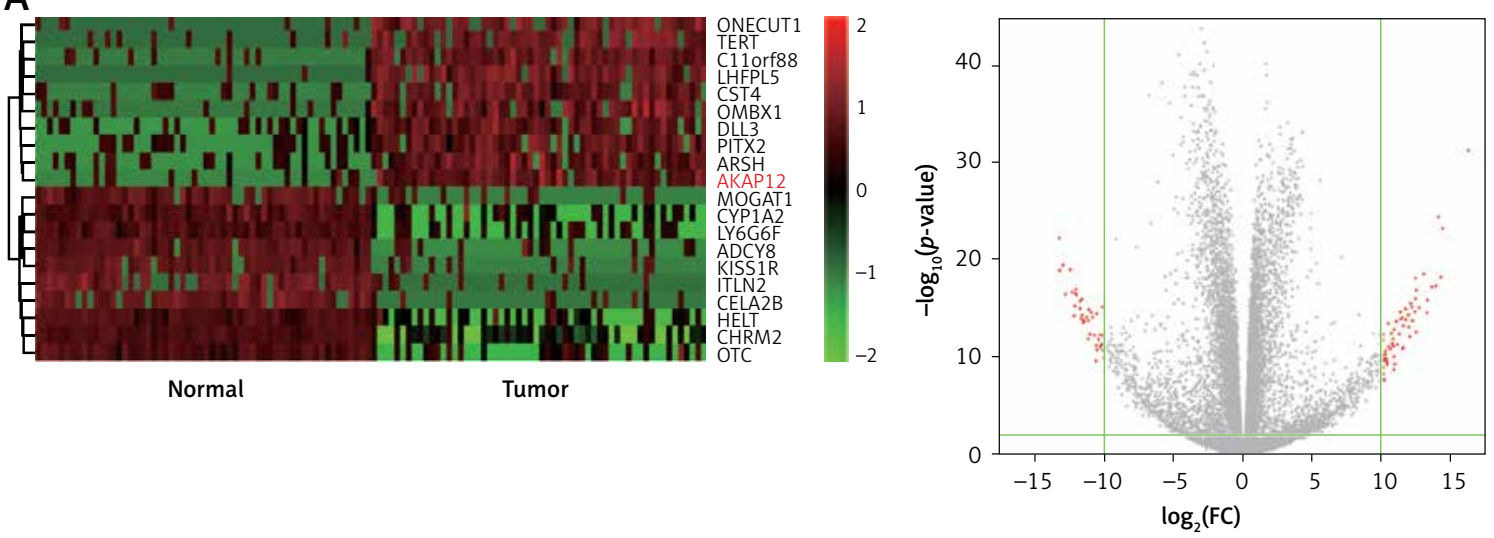

B
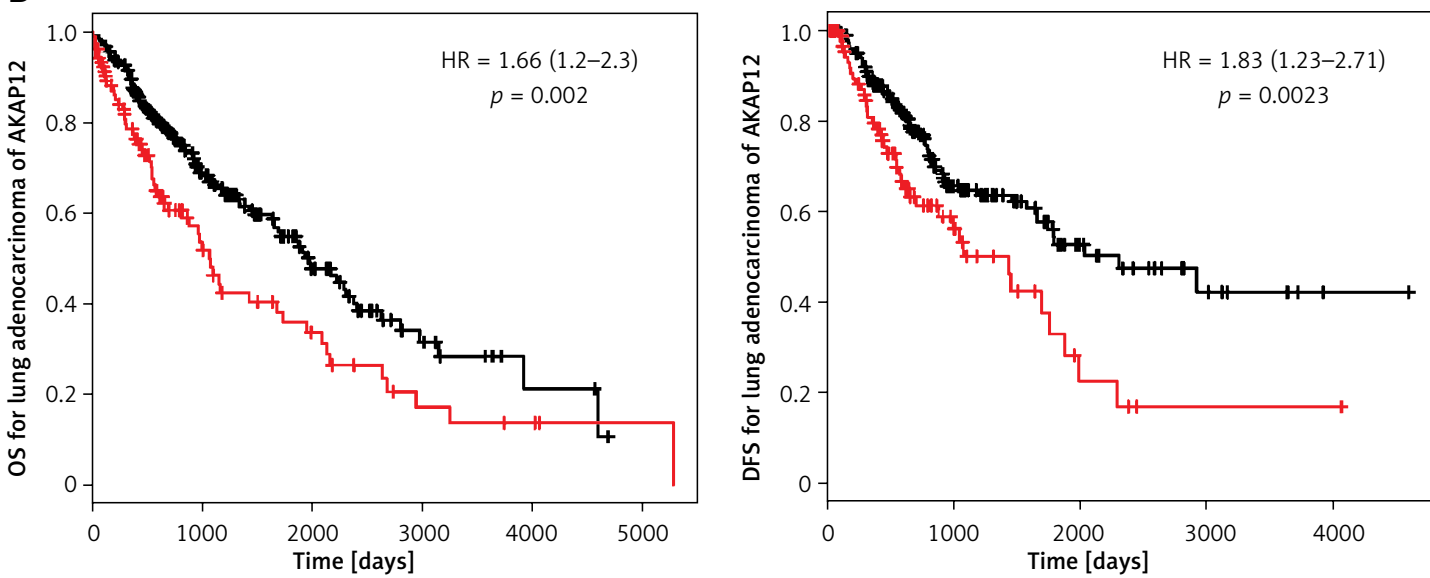

Number at risk

\begin{tabular}{|c|c|c|c|c|}
\hline 281 & 106 & 38 & 12 & 3 \\
\hline 104 & 30 & 14 & 5 & 3 \\
\hline
\end{tabular}

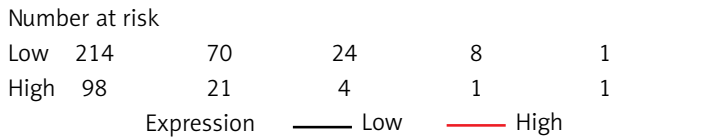

C

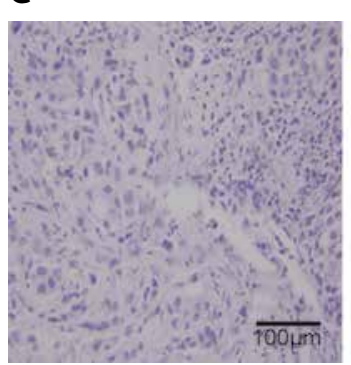

Adjacent

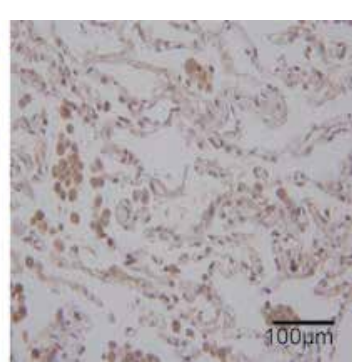

Tumor

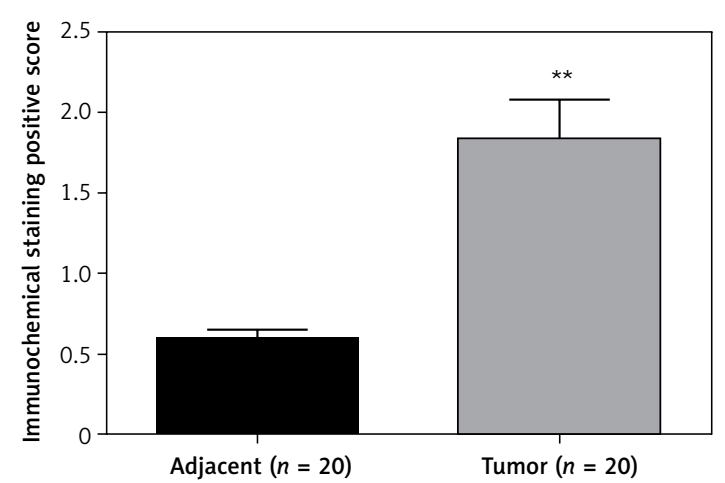

Figure 1. AKAP12 was highly expressed in lung adenocarcinoma tissues. A - Heat map and volcano plot for 20 genes in lung adenocarcinoma. AKAP12 was highly expressed in tumor tissues. B - AKAP12 high expression resulted in worse prognosis. Low expression of $A K A P 12$ significantly lengthened the overall survival time (OS) and disease-free survival time (DFS). C - Immunohistochemistry chart for AKAP12. AKAP12 was highly expressed in tumor tissues compared with adjacent ones regarding immunochemical staining positive score

mentary Table SIII and the AKAP12 staining value was higher in tumor tissues (average score $=1.84$ ) compared with normal tissues (average score $=$ 0.60) (Figure $1 \mathrm{C}$ ). The aberrantly high expression of AKAP12 could be a potential factor to promote the development of lung adenocarcinoma.
Up-regulation of $A K A P 12$ deteriorated lung adenocarcinoma in functional experiments

To further investigate the role of AKAP12 in lung adenocarcinoma, we utilized qRT-PCR and Western blot assay and confirmed that both the 
mRNA and protein level of AKAP12 were higher in cancer cell lines A549 and H1299 compared to the normal cell line HBE (all $p<0.01$, Figure $2 \mathrm{~A}$ ). Transient transfection was performed in both A549 and HBE cells using pcDNA3.1 vector, pcDNA3.1-AKAP12 cDNA or pcDNA3.1-AKAP12 shRNA. At $48 \mathrm{~h}$ after transfection, cells were collected and the biological functions were detected. According to the clone formation assay, cell proliferation was significantly upregulated in the AKAP12 group while it was downregulated in the AKAP12 shRNA group (Figure 2 B). Also, cell viability detected by MTT assay in A549 and H1299 cells with overexpression of AKAP12 both displayed an increase (all $p<0.05$, Figures 2 C, D). Therefore, AKAP12 overexpression could significantly promote cell proliferation and cell viability, and vice versa. As shown in Figure $3 \mathrm{~A}, A K A P 12$ overexpression could dramatically accelerate cell invasion while its suppression could inhibit cell invasion (all $p<0.01$ ). The wound healing assay also displayed a similar trend: at the $24^{\text {th }} \mathrm{h}$ after the scratch, more migration appeared in the AKAP12 overexpression group but less migration in the AKAP12 suppression group ( $p<0.01, p<0.001$, Figure $3 \mathrm{~B})$. As expected, the cell apoptosis rate was significantly downregulated in the AKAP12 group and was upregulated in the $A K A P 12$ shRNA group, confirming that AKAP12 suppressed cell apoptosis and led to deterioration of lung adenocarcinoma $(p<0.05$, $p<0.01, p<0.001$, Figure $3 \mathrm{C}$ ).

AKAP12 was the target of miR-338-3p and could be down-regulated by miR-338-3p

By TargetScan 7.0 and miRNA base selection, four miRNAs significantly regulated AKAP12 in terms of bioinformatics. MiR-107 directly targeted site 57 , miR-206 site 520, miR-613 site 522, and miR-338-3p site 864 (Figures 4 A, B). Different effects were judged from mirSVR score, PhastCons score, and context++ score percentile as suggested in Supplementary Table SII. MiR-338-3p had the lowest mirSVR score and a relatively high PhastCons score, indicating the highest thermal stability and a relatively good conservativeness. MiR-338-3p also had the highest context++ score, suggesting the greatest conservation and mRNA destabilization. Therefore, miR-338-3p could be the most suitable candidate for the target gene. Also, AKAP12 structure mutations were predicted according to the RNA Folding Form website, which showed the most significant changes in the miR338-3p group (Figure $4 \mathrm{C}$ and Supplementary Figures S1-S4). After transfection of $100 \mathrm{nM}$ miRNAs into HEK 293T cells, different effects of four miRNAs on AKAP12 expression were confirmed in Figure $4 \mathrm{D}$ by qRT-PCR detection, and miR-338-3p resulted in the most significant decrease in AKAP12
mRNA expression (both $p<0.01$ ). Scores, structure changes and mRNA level changes of AKAP12 combined confirmed that miR-338-3p could drastically downregulate AKAP12 expression and miR338-3p was selected as a topic. Then, luciferase activity of the control group, NC mimic group and miR-338-3p mimic group revealed that miR-338$3 p$ directly targeted $A K A P 12$ and downregulated its expression (Figure $4 \mathrm{E}$ ).

\section{MiR-338-3p could inhibit lung adenocarcinoma by $A K A P 12$ suppression}

To further understand the effect of miR-338$3 p$ on AKAP12 expression, cells transfected with different plasmids or sequences were divided into four groups, i.e. the control group, AKAP12 group, miR-338-3p mimic group and AKAP12+ miR-338-3p mimic group. AKAP12 significantly upregulated $A K A P 12$ mRNA expression while miR338-3p downregulated it, but AKAP12 and miR338-3p combined caused no significant differences $(p<0.05$, Figure $5 A$ ). AKAP12 protein expression was upregulated in the AKAP12 group and downregulated in the miR-338-3p group, but showed no significant differences in the AKAP12 + miR-338$3 p$ mimic group ( $p<0.05$, Figure $5 \mathrm{~B}$ ). Also, after dividing the patients into two groups according to the median miR-338-3p expression, we found that high expression of miR-338-3p would improve the prognosis, considering the higher DFS and OS rates (Figures 5 C, D). With the Kaplan-Meier method and Cox regression models, univariate and multivariate analysis for the overall survival of lung adenocarcinoma was performed using the data from the TCGA database (Tables II and III). Stage event pathologic stage (I-IV), M stage, miR388-3p and AKAP12 expression were all correlated with the prognosis in univariate analysis while both miR-388-3p and AKAP12 expression were recognized as independent prognostic factors for the overall survival. The above results verified that miR-338-3p suppressed the progression of lung adenocarcinoma by inhibiting AKAP12.

\section{Discussion}

Our study indicated that the expression level of AKAP12 was high in lung adenocarcinoma. Meanwhile, high expression of $A K A P 12$ predicted a poor prognosis. AKAP12 alpha previously was reported to be a promoter in lung cancer in the research of Jo et al.; AKAP12 $\alpha$ protein was downregulated in H358, H460 and Calu-1 cells but not H1299 cells, which to a degree is consistent with this study. The methylation status of $5^{\prime} \mathrm{CpG}$ islands of the $A K A P 12 \alpha$ gene in lung tissues might be the reason for the promotion [17]. AKAP12, which was methylated at nearly $40 \%$ prevalence in lung ade- 

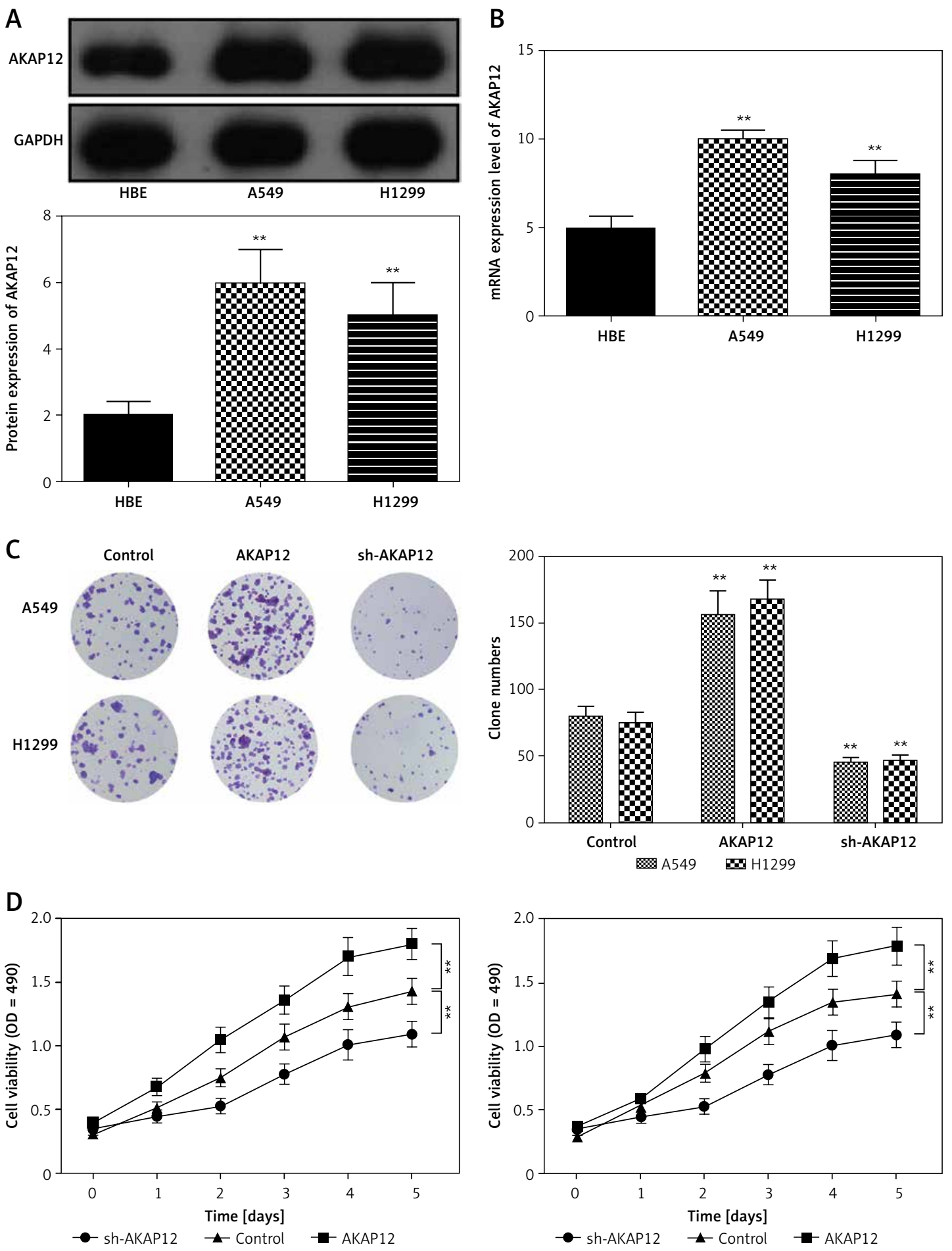

Figure 2. AKAP12 was highly expressed in lung adenocarcinoma cells. A - AKAP12 was highly expressed in A549 and H1299 cells in protein level. B - AKAP12 was highly expressed in A549 and H1299 cells in mRNA level. C - Clone formation revealed that AKAP12 could promote A549 and H1299 cell proliferation and its knockdown could inhibit proliferation. D - MTT assay revealed that AKAP12 significantly increased cell viability while shAKAP12 decreased cell viability

${ }^{* *} p<0.01$, compared with control group.

nocarcinoma samples and modulates the expression of genes involved in the cell cycle and apoptosis, was not associated with age at diagnosis or stage of lung cancer [22]. This study by contrast proved a relationship between AKAP12 and DFS as well as OS, which hallmarked its biomarker role in lung adenocarcinoma prognosis. After manipulating the expression of AKAP12 in A549 and 

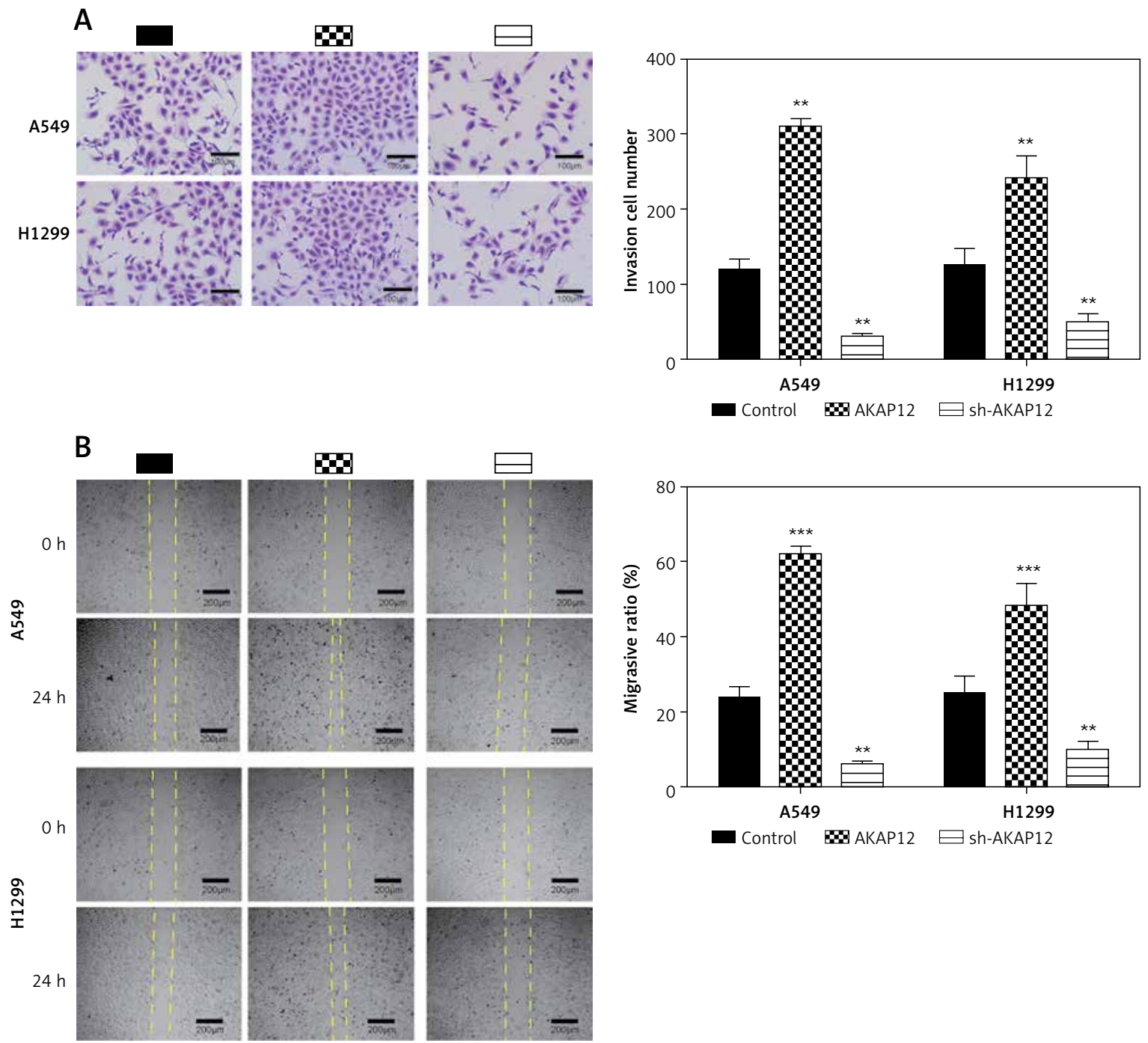

\section{c}
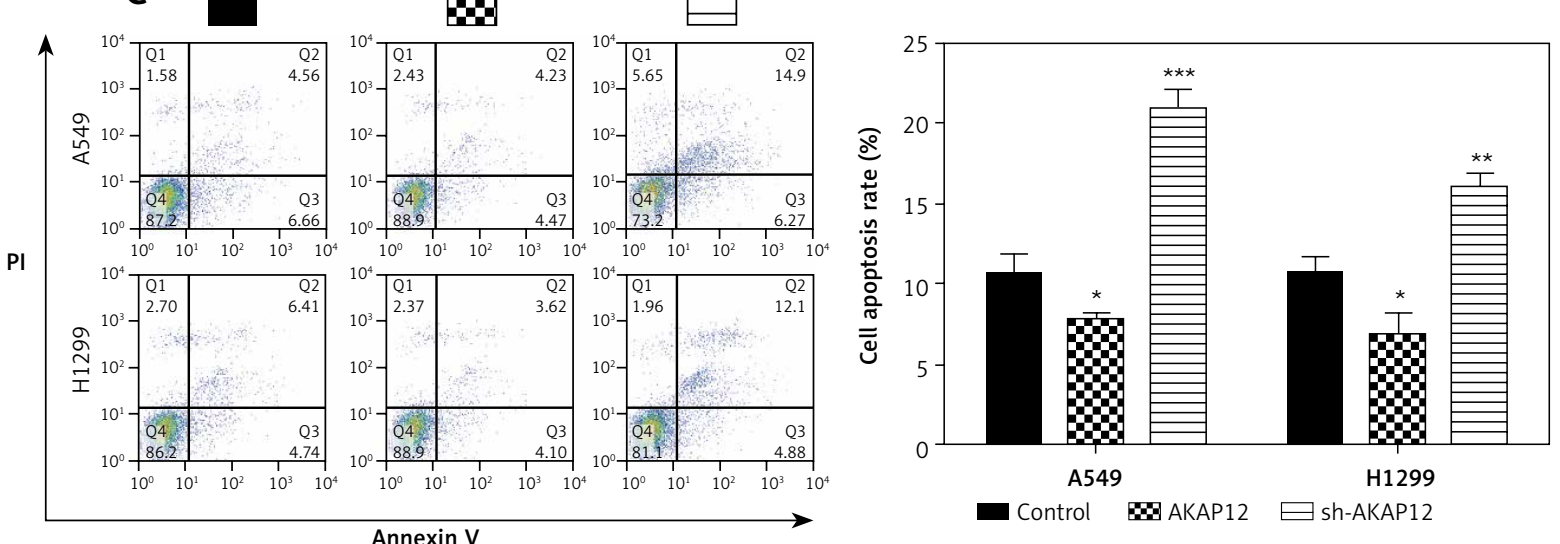

Figure 3. AKAP12 deteriorated lung adenocarcinoma in functional experiments. $\mathbf{A}-$ Transwell assay revealed that AKAP12 could increase cell invasion and its knockdown could inhibit the process. B - Wound healing assay revealed that $A K A P 12$ could improve migration ratio and its knockdown could decrease the ratio. $\mathrm{C}$ - Flow cytometry revealed that AKAP12 could reduce cell apoptosis rate and its knockdown could increase cell apoptosis rate

${ }^{*} p<0.05,{ }^{* *} p<0.01,{ }^{* * *} p<0.001$, compared with control group. 
A

AKAP12 3'UTR

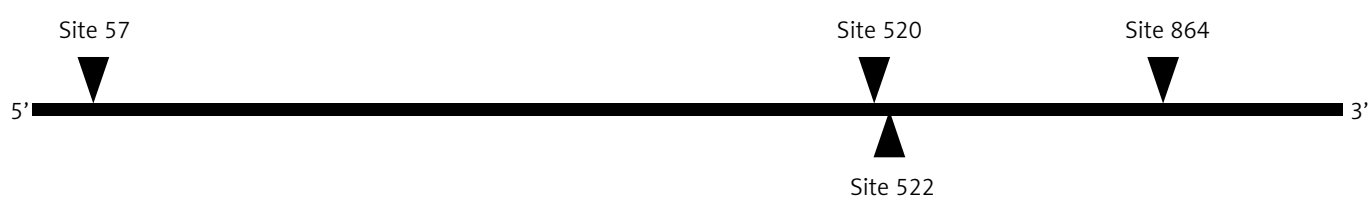

B

Wild site 57

has-miR-107

Mutant site 57 (M1)

Wild site 522

has-miR-613

Mutant site 522 (M3)

C

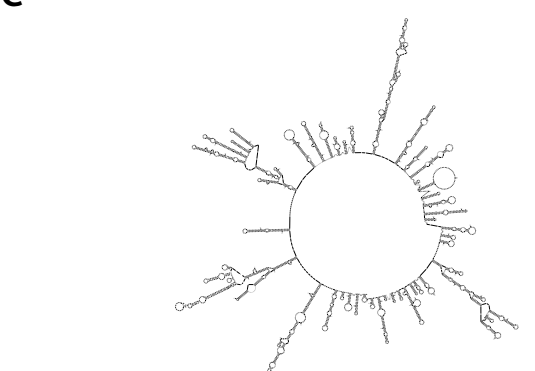

WT

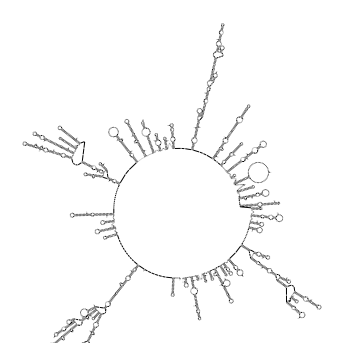

M1
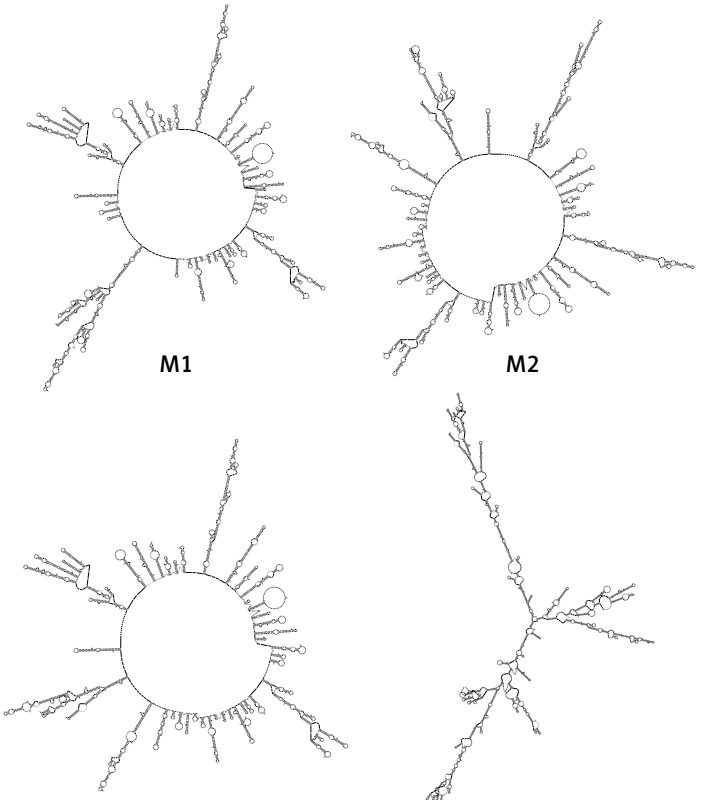

M3
M1

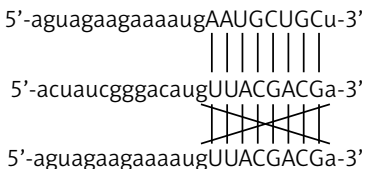

M3
M4
Wild site 520

has-miR-206

Mutant site 520 (M2)

Wild site 864

has-miR-338-3p

Mutant site 864 (M4)
$\mathrm{M} 2$

5'-gccuccuagaugUAACAUUCCu-3' \|\|\|\| ggugugugaaggAAUGUAAGGu-3' WH 5'-gccuccuagaugAAUGUAAGGu-3'

M4

5'-gauucuACUCUUAUAUGCUGGa-3'<smiles>C1=CC=C1</smiles><smiles></smiles>

5'-guuguuUUAGUGACUACGACCu-3'

YHY

5'-gauucuUUAGUGACUACGACCa-3'

D

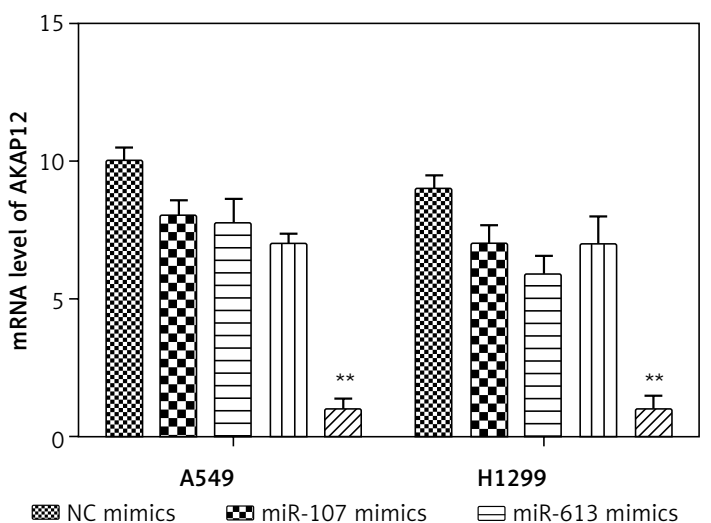

س miR-206 mimics 20 miR-338-3p mimics

E

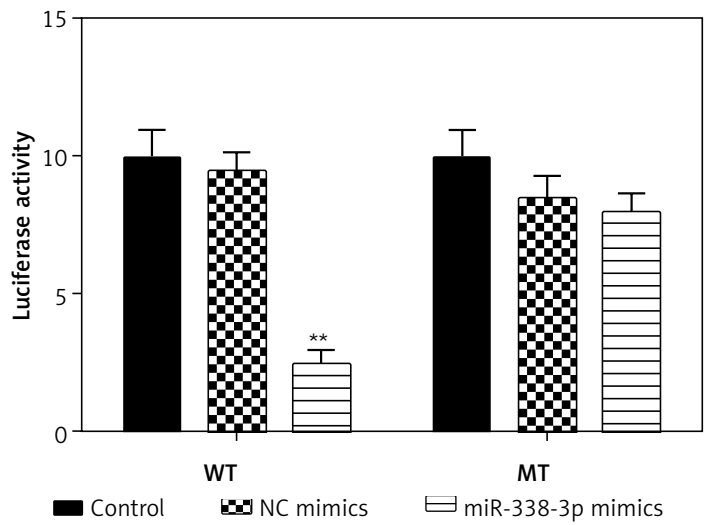

Figure 4. AKAP12 was the target of miR-338-3p and could be down-regulated by miR-338-3p. A - Targeted sites of four miRNAs are displayed. MiR-107 targeted at site 57, miR-206 at site 520, miR-613 at site 522, and miR-338-3p at site 864. B - Four miRNAs all directly targeted at AKAP12 instead of its variants. C - Structures changes after miRNAs combination according to RNA folding form 23.0. AKAP12 mutant 4 had significant structure changes caused by miR-338-3p. D - Q-PCR of four miRNAs revealed that four miRNAs could downregulate AKAP12 expression, and miR-338-3p displayed the most significant changes. E - MiR-338-3p could inhibit the luciferase activity of WT 3'UTR of AKAP12 but had little influence on MT 3'UTR of AKAP12

${ }^{* *} p<0.01$, compared with control group. 
A

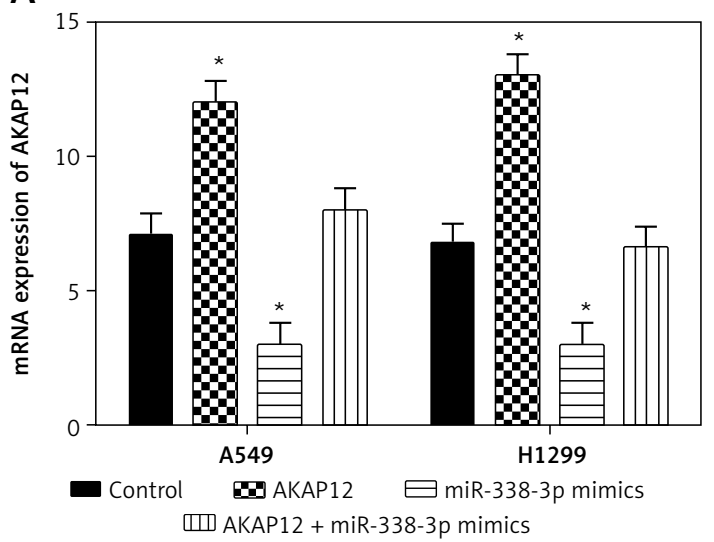

C

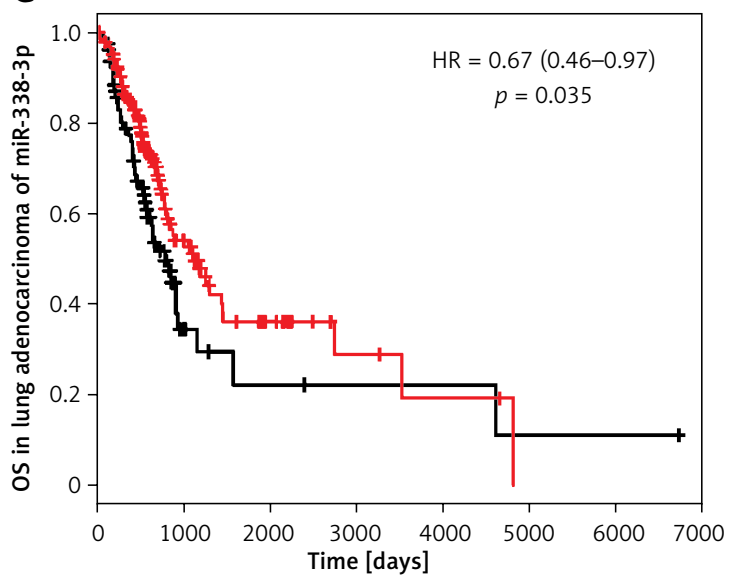

Number at risk

$\begin{array}{lllllllll}\text { Low } & 80 & 8 & 3 & 2 & 2 & 1 & 1 & 0\end{array}$

High $\begin{array}{llll}190 & 38 & 13 & 4\end{array}$

Expression
B
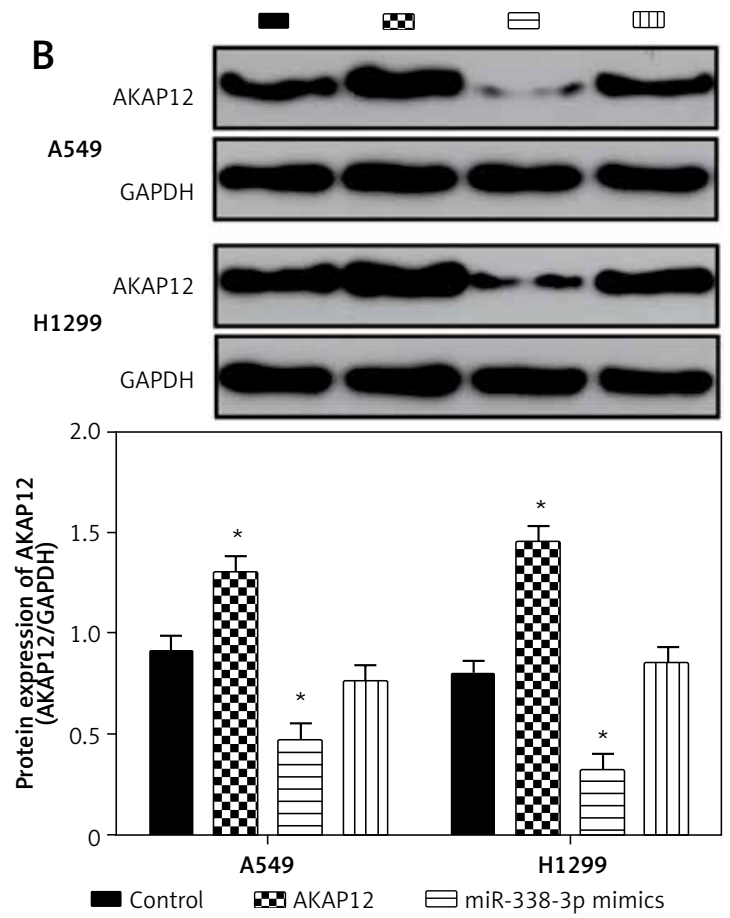

D

س AKAP12 + miR-338-3p mimics

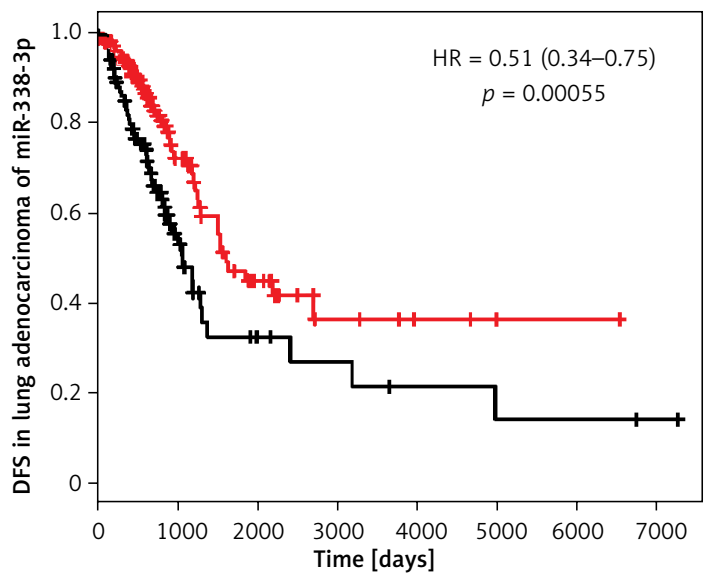

Number at risk

Low 106

High 209

Figure 5. MiR-338-3p inhibited lung adenocarcinoma by AKAP12 suppression. A, B - MiR-338-3p could down-regulate the expression of AKAP12 according to qRT-PCR and western blot. C, D - MiR-338-3p high expression led to better DFS and OS

${ }^{*} p<0.05$, compared with control group.

$\mathrm{H} 1299$ cells, we confirmed that AKAP12 acts as an oncogene and facilitated the proliferation, migration and invasion of lung cancer cells.

The promotion effect of AKAP12 on lung adenocarcinoma was associated with miR-338-3p as we proved the target relationship between miR338-3p and AKAP12. MiR-338 was reported to abate lung cancer in vitro and in vivo by targeting ITGB3 [13], a conclusion similar to this study. MiR338-3p could suppress lung cancer tumorigenesis and metastasis in vitro and in vivo by targeting the EMT regulator Sox4 as well [9]. MiR-338-3p was also predicted in an integrative bioinformatics analysis as a biomarker for diagnosis of NSCLC adenocarcinoma [23]. In this research, miR-338$3 p$ was discovered to target AKAP12 and inhibited lung adenocarcinoma development, which to our knowledge had not been studied before. As a result, our study demonstrated a new regulatory mechanism of miR-338-3p, which revealed a better strategy for lung adenocarcinoma treatment.

However, there are some limitations in this study. The underlying mechanisms of AKAP12 regulation in lung adenocarcinoma remains to be 
Table II. Univariate analysis for overall survival

\begin{tabular}{|c|c|c|c|}
\hline Factors & $\beta$ & $\mathrm{HR}(95 \% \mathrm{Cl}$ for HR) & $P$-value \\
\hline Age & -0.03964 & $0.9611(0.7727-1.196)$ & 0.7218 \\
\hline Gender & 0.04459 & $1.046(0.8558-1.278)$ & 0.6626 \\
\hline Location in lung parenchyma & -0.3242 & $0.7231(0.5042-1.037)$ & 0.07671 \\
\hline Targeted molecular therapy & 0.05719 & $1.059(0.6697-1.674)$ & 0.8067 \\
\hline Radiation therapy & -0.04471 & $0.9563(0.4826-1.895)$ & 0.898 \\
\hline Stage event pathologic stage & -0.3167 & $0.7285(0.5545-0.9573)$ & $0.02244^{*}$ \\
\hline T stage & 0.0185 & $1.019(0.7429-1.397)$ & 0.9085 \\
\hline $\mathrm{N}$ stage & -0.2032 & $0.8161(0.6086-1.094)$ & 0.1739 \\
\hline M stage & -0.3755 & $0.687(0.5525-0.8541)$ & $0.000679^{*}$ \\
\hline Tobacco smoking history & -0.07018 & $0.9322(0.7598-1.144)$ & 0.5011 \\
\hline miR-338-3p & 0.2738 & $1.315(1.051-1.645)$ & $0.01636^{*}$ \\
\hline AKAP12 & -0.2867 & $0.7507(0.6032-0.9344)$ & $0.009983^{*}$ \\
\hline
\end{tabular}

$H R$ - hazard radio for death. Stage event pathologic stage includes stages I-IV. ${ }^{*} p<0.05$ indicates statistical significance.

Table III. Multivariate analysis for overall survival of AKAP12 and miR-338

\begin{tabular}{|lcc|}
\hline Factors & HR $(95 \% \mathrm{Cl}$ for HR) & $P$-value \\
\hline $\begin{array}{l}\text { Stage event } \\
\text { pathologic stage }\end{array}$ & $0.781(0.5917-1.0308)$ & 0.0809 \\
\hline M stage & $0.6805(0.5446-0.8502)$ & $7.00 \mathrm{E}-04$ \\
\hline SELE & $1.4344(1.1398-1.8052)$ & 0.0021 \\
\hline TLX2 & $0.7101(0.5682-0.8876)$ & 0.0026 \\
\hline
\end{tabular}

$H R$ - hazard radio for death. Stage event pathologic stage included stages I-IV. * $p<0.001$ indicates statistical significance.

further investigated including methylation and phosphorylation. AKAP $12 \alpha$ and AKAP $12 \beta$ expression and corresponding results still need exploration as well as in the case of opposite results when it comes to lung adenocarcinoma. MiRNAs which may have better combination with AKAP12 could be discussed in future studies for the consideration of better application in lung adenocarcinoma treatment.

In conclusion, AKAP12 was upregulated in lung adenocarcinoma, and its high expression promoted tumor development and predicted poor prognosis. High expression of AKAP12 in lung adenocarcinoma led to proliferation, migration and invasion of cancer cells, and apoptosis inhibition. MiR-338-3p directly targeted AKAP12 and inhibited lung adenocarcinoma by $A K A P 12$ restraint. The regulation of miR-338-3p and AKAP12 could be of value for clinic targeted therapy of lung adenocarcinoma.

\section{Acknowledgments}

This study was supported by Science and Technology Project of Health and Family Planning Commission of Hebei (No. ZD20140161).

\section{Conflict of interest}

The authors declare no conflict of interest.

\section{References}

1. Siegel RL, Miller KD, Jemal A. Cancer statistics, 2016. CA Cancer J Clin 2016; 66: 7-30.

2. Wu KL, Tsai MJ, Yang CJ, et al. Liver metastasis predicts poorer prognosis in stage IV lung adenocarcinoma pa-

tients receiving first-line gefitinib. Lung Cancer 2015; 88: 187-94.

3. Siegel RL, Miller KD, Jemal A. Cancer statistics, 2017. CA Cancer J Clin 2017; 67: 7-30.

4. de Langen AJ, Smit EF. Therapeutic approach to treating patients with BRAF-mutant lung cancer: latest evidence and clinical implications. Ther Adv Med Oncol 2017; 9: 46-58.

5. Lujambio A, Lowe SW. The microcosmos of cancer. Nature 2012; 482: 347-55.

6. Krol J, Loedige I, Filipowicz W. The widespread regulation of microRNA biogenesis, function and decay. Nat Rev Genet 2010; 11: 597-610.

7. Kos A, Olde Loohuis NF, Wieczorek ML, et al. A potential regulatory role for intronic microRNA-338-3p for its host gene encoding apoptosis-associated tyrosine kinase. PLoS One 2012; 7: e31022.

8. Wang G, Sun Y, He Y, Ji C, Hu B, Sun Y. MicroRNA-338-3p inhibits cell proliferation in hepatocellular carcinoma by target forkhead box P4 (FOXP4). Int J Clin Exp Pathol 2015; 8: 337-44. 
9. Jin $\mathrm{Y}$, Zhao M, Xie Q, Zhang H, Wang Q, Ma Q. MicroRNA-338-3p functions as tumor suppressor in breast cancer by targeting SOX4. Int J Oncol 2015; 47: 1594602.

10. Zhang P, Shao G, Lin X, Liu Y, Yang Z. MiR-338-3p inhibits the growth and invasion of non-small cell lung cancer cells by targeting IRS2. Am J Cancer Res 2017; 7: 53-63.

11. Sun J, Feng X, Gao S, Xiao Z. microRNA-338-3p functions as a tumor suppressor in human nonsmallcell lung carcinoma and targets Ras-related protein 14. Mol Med Rep 2015; 11: 1400-6.

12. Hong-Yuan W, Xiao-Ping C. miR-338-3p suppresses epithelial-mesenchymal transition and metastasis in human nonsmall cell lung cancer. Indian J Cancer 2015; 52 Suppl 3: E168-71.

13. Chen X, Wei L, Zhao S. miR-338 inhibits the metastasis of lung cancer by targeting integrin beta3. Oncol Rep 2016; 36: 1467-74.

14. Zhang G, Zheng H, Zhang G, et al. MicroRNA-338-3p suppresses cell proliferation and induces apoptosis of non-small-cell lung cancer by targeting sphingosine kinase 2. Cancer Cell Int 2017; 17: 46.

15. Li Y, Chen P, Zu L, Liu B, Wang M, Zhou Q. Micro-RNA338-3p suppresses metastasis of lung cancer cells by targeting the EMT regulator Sox4. Am J Cancer Res 2016; 6: 127-40.

16. Hayashi $M$, Nomoto $S$, Kanda $M$, et al. Identification of the A kinase anchor protein 12 (AKAP12) gene as a candidate tumor suppressor of hepatocellular carcinoma. J Surg Oncol 2012; 105: 381-6.

17. Jo UH, Whang YM, Sung JS, Kim YH. Methylation of AKAP12 alpha\} promoter in lung cancer. Anticancer Res 2010; 30: 4595-600.

18. Xia W, Ni J, Zhuang J, Qian L, Wang P, Wang J. MiR-103 regulates hepatocellular carcinoma growth by targeting AKAP12. Int J Biochem Cell Biol 2016; 71: 1-11.

19. Bateman NW, Jaworski E, Ao W, et al. Elevated AKAP12 in paclitaxel-resistant serous ovarian cancer cells is prognostic and predictive of poor survival in patients. J Proteome Res 2015; 14: 1900-10.

20. Finger EC, Castellini L, Rankin EB, et al. Hypoxic induction of AKAP12 variant 2 shifts PKA-mediated protein phosphorylation to enhance migration and metastasis of melanoma cells. Proc Natl Acad Sci USA 2015; 112: $4441-6$.

21. Riffo-Campos AL, Riquelme I, Brebi-Mieville P. Tools for sequence-based miRNA target prediction: what to choose? Int J Mol Sci 2016; 17. pii: E1987.

22. Tessema M, Belinsky SA. Mining the epigenome for methylated genes in lung cancer. Proc Am Thorac Soc 2008; 5: 806-10.

23. Shao Y, Liang B, Long F, Jiang SJ. Diagnostic microRNA biomarker discovery for non-small-cell lung cancer adenocarcinoma by integrative bioinformatics analysis. Biomed Res Int 2017; 2017: 2563085. 